\title{
Utilization of Poly(ADP-Ribose) Polymerase Inhibitors in Ovarian Cancer: A Retrospective Cohort Study of US Healthcare Claims Data
}

\author{
Rebecca C. Arend (1) - David M. O'Malley (1) - Susana Banerjee (1) · \\ Kimmie McLaurin • Richard Davidson • Gráinne H. Long (1)
}

Received: September 3, 2021 / Accepted: October 8, 2021 / Published online: November 2, 2021

(C) The Author(s) 2021

\begin{abstract}
Introduction: We aimed to characterize realworld utilization of poly(ADP-ribose) polymerase (PARP) inhibitors (PARPi) in women with ovarian cancer (OC).

Methods: This retrospective observational study of claims data from US MarketScan ${ }^{\circledR}$ Commercial/Medicare Supplemental databases
\end{abstract}

Rebecca C. Arend and David M. O'Malley contributed equally to this work.

Supplementary Information The online version contains supplementary material available at https:// doi.org/10.1007/s12325-021-01959-5.

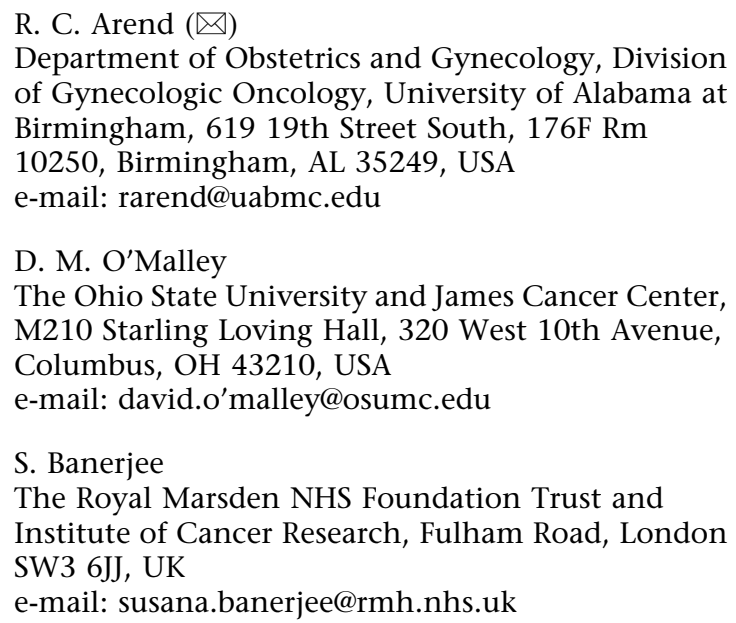
of Gynecologic Oncology, University of Alabama at Birmingham, 619 19th Street South, 176F Rm 10250, Birmingham, AL 35249, USA

e-mail: rarend@uabmc.edu

D. M. O’Malley

The Ohio State University and James Cancer Center, M210 Starling Loving Hall, 320 West 10th Avenue, Columbus, OH 43210, USA

e-mail: david.o'malley@osumc.edu

S. Banerjee

The Royal Marsden NHS Foundation Trust and Institute of Cancer Research, Fulham Road, London SW3 6JJ, UK

e-mail: susana.banerjee@rmh.nhs.uk

included women with OC initiating olaparib, niraparib, or rucaparib from January 1, 2017, to May 31, 2019. Patients were observed from first outpatient prescription until at least 30 days' follow-up. Clinical events of interest (CEIs), based on adverse reactions in PARPi prescribing information, were identified from claims using ICD-9/10 codes. Other outcomes included dose modification, persistence, adherence, healthcare resource utilization (HCRU), and cost.

Results: Overall, 303, 348, and 162 women with OC received olaparib, niraparib, and rucaparib, respectively. During follow-up, risk of any CEI was higher with niraparib versus olaparib (odds ratio $3.36[95 \%$ confidence interval

\author{
K. McLaurin \\ AstraZeneca Pharmaceuticals LP, One MedImmune \\ Way, Gaithersburg, MD 20878, USA \\ e-mail: kimmie.mclaurin@astrazeneca.com \\ R. Davidson · G. H. Long \\ AstraZeneca Pharmaceuticals LP, City House, 136 \\ Hills Road, Cambridge CB2 8PA, UKR. Davidson \\ e-mail: richard.davidson@astrazeneca.com \\ G. H. Long \\ e-mail: grainne.long@astrazeneca.com
}


2.00-5.65]) and niraparib versus rucaparib (2.09 [1.10-3.95]), with no significant difference between rucaparib and olaparib (1.61 [0.93-2.79]). PARPi dose decreases were observed in $21.1 \%, 35.1 \%$, and $30.2 \%$ of olaparib-, niraparib-, and rucaparib-treated patients, respectively. Persistence (no treatment gaps of more than 90 days) was significantly higher $(P<0.05)$ with olaparib $(62.2 \%)$ versus niraparib (35.9\%) and rucaparib (48.7\%); adherence (medication possession ratio, MPR $\geq 80 \%)$ was $80.2 \%$ versus $38.6 \%$ and $63.2 \%$, respectively $(P<0.001)$. Inpatient admissions and outpatient service use were higher with niraparib and rucaparib versus olaparib, reflected in mean ( \pm standard deviation) total medical costs (excluding pharmacy) of $\$ 5393 \pm 8828$ for olaparib, $\$ 7732 \pm 14,054$ for niraparib, and $\$ 6868 \pm 7929$ for rucaparib.

Conclusion: Differences between the licensed PARPi were observed in the risk of experiencing a CEI, likelihood of dose modifications, ability to receive continuous PARPi therapy, HCRU, and costs.

Keywords: Ovarian cancer; Real-world evidence; PARP inhibitors; Tolerability; Dose modification; Healthcare resource utilization

\section{Key Summary Points}

Why carry out this study?

A better understanding of the real-world tolerability, patient adherence and persistence, and associated healthcare resource utilization and costs of available PARP inhibitor therapies for ovarian cancer is needed

This retrospective observational study characterized real-world utilization of poly(ADP-ribose) polymerase (PARP) inhibitors in women with ovarian cancer

\section{What was learned from this study?}

Differences were observed in the risk of experiencing clinical events of interest between licensed PARP inhibitors
Differences in likelihood of dose modifications, ability to receive continuous therapy, healthcare resource utilization, and cost were also identified

This study will help inform physicians and payers of the importance of considering the respective profiles of PARP inhibitors when selecting the most appropriate maintenance therapy for patients with ovarian cancer

\section{INTRODUCTION}

The introduction of targeted therapies such as poly(ADP-ribose) polymerase (PARP) inhibitors (PARPi) has transformed the treatment of ovarian cancer (OC) $[1,2]$. Three PARPi (olaparib, niraparib, and rucaparib) are currently approved for the treatment of OC, and while they share a common mechanism of action, each has a different chemical structure, as well as unique pharmacodynamic and pharmacokinetic properties [3, 4]. Several differences between individual PARPi have been characterized at the cellular level, including selectivity towards different members of the PARP family, differences in DNA strand break repair, cell cycle arrest, and antiproliferative activities [5]. At the clinical level, these properties manifest as differences in potency, efficacy, safety, and off-target effects [4, 6-8]; consequently, each PARPi also has a different dosing schedule [9-11].

Olaparib was the first PARPi approved for the treatment of breast cancer gene (BRCA)-mutated advanced OC in 2014. Since then, olaparib, niraparib, and rucaparib have been approved, regardless of biomarker status, for maintenance treatment following complete or partial response to platinum-based therapy in platinum-sensitive recurrent OC [9-11]. Olaparib and niraparib received approval for this indication in 2017, with approval for rucaparib following in 2018 [12]. More recent randomized controlled trials (RCTs) have led to US Food and Drug Administration (FDA) approval of frontline PARPi maintenance therapy $[9,11,13,14]$. Furthermore, PARPi are also approved as 
monotherapy for women with recurrent $B R C A$ mutated (after two or more prior lines [rucaparib] or three or more prior lines [olaparib]) or homologous recombination repair deficient (HRD; after three or more prior lines [niraparib]) tumors [9-12]. See Supplementary Table 1 for details of US FDA PARPi approval dates and indications.

In pivotal PARPi RCTs, the most common adverse events (AEs) that led to dose modifications were hematologic toxicities; other common AEs associated with this class of therapy include gastrointestinal disorders, photosensitivity, fatigue, and infection [15-17]. RCT data suggest differences between PARPi in terms of tolerability and dose modifications [15-18]. Real-world evidence of PARPi use in clinical practice reported a safety profile largely consistent with RCT data [19-24], with emerging data supporting distinct tolerability profiles for each PARPi [25]. Tolerability is likely to be an important factor for long-term PARPi maintenance therapy. A better understanding of realworld patient adherence (the extent to which patients act in accordance with the prescribed interval and dose of a regimen) and persistence (the duration of time from initiation to discontinuation of therapy) with PARPi is needed [26], alongside associated healthcare resource utilization (HCRU), i.e., all-cause inpatient, outpatient, and pharmaceutical utilization and costs.

Here we characterized tolerability (in terms of clinical events of interest [CEIs]), dose modification, treatment adherence and persistence, and the HCRU profile of real-world PARPi use in maintenance and treatment settings in a large population of women with OC in the USA.

\section{METHODS}

\section{Setting}

This study was a longitudinal, retrospective, cohort analysis of data from the US MarketScan ${ }^{\circledR}$ Commercial and Medicare Supplemental Databases [27]. These databases contain healthcare data from patients insured commercially or as part of the national Medicare program, including detailed cost, use, and outcomes data for healthcare services performed in both inpatient and outpatient settings. Data have been anonymized and comply with the Health Insurance Profitability and Accountability Act. As the patient and provider data included in these analyses were fully de-identified, this study was exempt from institutional review board approval.

\section{Study Population}

Eligible patients were adult (aged 18 years or more) women diagnosed with epithelial ovarian, fallopian tube, or primary peritoneal cancer between January 1, 2015, and May 31, 2019, who were first prescribed a PARPi (olaparib, niraparib, or rucaparib) between January 1, 2017, and May 31, 2019 (index period; Supplementary Fig. 1).

Diagnosis was based on International Classification of Diseases (ICD) 9/10 code criteria (Supplementary Table 2). Patients were identified on the basis of having at least one inpatient claim or two non-diagnostic outpatient claims 30-365 days apart. The date the patient first received olaparib, niraparib, or rucaparib within the index period (January 1, 2017, to May 31, 2019) was classed as the index date; baseline refers to the 6-month period prior to the index date. Patients were required to have at least 6 months' enrollment (baseline) prior to, and at least 30 days after, the index date and were followed up until end of observation period (May 31, 2019), disenrollment, or death, whichever occurred first. Patients initiating PARPi prior to January 1, 2017, and those without an OC diagnosis within 6 months of the index date were excluded.

\section{Study Outcomes}

Sixteen predefined CEIs were selected for evaluation based on the adverse reactions documented in the PARPi prescribing information: acute myeloid leukemia (AML)/myelodysplastic syndromes (MDS), anemia, leukopenia/neutropenia, thrombocytopenia, acute kidney injury, arthralgia, constipation, diarrhea, 
nausea/vomiting, dermatitis/rash/photosensitivity, fatigue, hypertension, infection, insomnia, pneumonitis, and transaminitis [9-11]. CEIs were identified from the presence of ICD-9CM or ICD-10-CM codes.

Treatment duration was defined as the total number of days' supply without a gap of 90 days or more [28]. Dose was calculated for each PARPi pharmacy claim as (quantity supplied $\times$ drug strength)/days' supply and was rounded to the nearest $50 \mathrm{mg}$. Highest indicated doses were taken from the prescribing information for each PARPi as 600, 800, 300, and $1200 \mathrm{mg} /$ day for olaparib tablets, olaparib capsules, niraparib capsules, and rucaparib tablets, respectively [9-11].

The methods used for measuring persistence and adherence from claims data have been described previously [28]. Persistence with the PARPi regimen was defined by no treatment gaps of more than 90 days during the 6-month period following initial PARPi use (index date) [28]. Time to non-persistence was calculated as the number of days that the patient was in possession of medication based on the days' supply of the PARPi in the fixed 6-month follow-up period. Medication possession ratio (MPR), a measure of adherence, was measured as the sum of days' supply divided by the 6-month (180-day) follow-up period; patients with MPR $<80 \%$ were categorized as non-adherent [28]. Persistence, days to non-persistence, and adherence (MPR $\geq 80 \%)$ to the PARPi regimen are reported for the subset of patients who had at least 6 months of continuous follow-up.

All-cause HCRU was measured during the baseline period and the period over which the index PARPi regimen was taken, for outpatient encounters (physician office, emergency room [ER] visit, and other outpatient services, including outpatient pharmacy infusions, radiation, and laboratory services) and for inpatient admissions. Healthcare costs were based on paid amounts of adjudicated claims, as well as patient cost sharing in the form of co-payment, deductibles, and co-insurance. All dollar estimates were adjusted for inflation using the Medical Care Component of the Consumer Price Index and standardized to the 2018 US dollar value (last full calendar year under study). To account for the variable-length follow-up period, rates of CEIs were described per persontime, and HCRU and healthcare costs are presented as per patient per month (PPPM).

\section{Statistical Analysis}

Participant characteristics at baseline were reported using mean (standard deviation [SD]), percentage (number), and/or median (interquartile range $[\mathrm{IQR}]$ ), depending on the distribution of underlying variables. Frequency of CEIs, dose modifications, adherence, and persistence, as well as all-cause HCRU and costs, were summarized by PARPi regimen. The first instance of a prespecified CEI is reported. Statistical comparisons were performed using chisquared or Fisher's exact tests for categorical variables and $t$ tests for continuous variables.

Whether experiencing a prespecified CEI varied between patients receiving the different PARPi was evaluated by logistic regression; odds ratios (ORs) and 95\% confidence intervals (CIs) by the Clopper-Pearson method are reported. Time to CEI, inpatient admissions, discontinuation, and dose decrease for different PARPi treatments were calculated by Cox proportional hazards regression. Time to event was measured from first PARPi initiation (index date) until CEI/discontinuation or the censor date (May 31, 2019), whichever occurred first. PARPi treatment during follow-up, National Cancer Institute (NCI)-adapted Charlson Comorbidity Index (CCI), bevacizumab use, and cancer-related surgery during baseline were considered a priori confounders and included in all models. To take into account the occurrence of relevant CEIs during the baseline period and ensure that the proportional hazards assumption was not violated, Cox proportional hazards regression models were stratified by the presence of the relevant baseline CEI [29]. Sensitivity analyses were performed on the basis of variations in starting dose and days' treatment gap, and among the subpopulation of patients who persisted with their PARPi regimen (see Supplementary Methods). Hazard ratios (HRs) and 95\% CIs are reported. Statistical significance was 
determined on the basis of $P<0.05$, which represents a nominal level of statistical significance.

Data extraction and descriptive analyses were conducted using WPS version 4.2 (World Programming, Romsey, UK). Multivariable models were generated with $\mathrm{R}$ version 3.6 ( $\mathrm{R}$ Foundation for Statistical Computing, Vienna, Austria).

\section{RESULTS}

\section{Baseline Characteristics}

Between January 1, 2017, and May 31, 2019, 303, 348, and 162 patients with OC received olaparib, niraparib, and rucaparib, respectively (Table 1). Of the patients who received olaparib, $27.1 \%, 41.6 \%$, and $31.4 \%$ initiated treatment in 2017,2018 , and 2019, respectively, whereas the majority of patients who received niraparib and rucaparib initiated treatment in 2017 or 2018. Median (IQR) follow-up for patients prescribed olaparib, niraparib, and rucaparib was 224.0 (293.0), 295.5 (339.0), and 265.0 (275.0) days, respectively.

Baseline demographics and clinical characteristics among the patients treated with a PARPi were similar, including mean age at diagnosis, evidence of chemotherapy, and proportion of patients commercially insured. The NCI-adapted CCI, a measure of burden of disease, was comparable across treatment groups (mean [SD] 0.7 [1.0], 0.9 [1.1], and 0.8 [1.1] for olaparib, niraparib and rucaparib, respectively).

\section{Clinical Events of Interest}

In the 6 months prior to initiating PARPi (baseline), the percentage of patients experiencing any hematologic or non-hematologic CEIs was generally consistent across groups (Supplementary Table 3).

Following PARPi initiation, the percentage of patients who experienced any CEI during follow-up was $81.5 \% \quad(n=247 / 303), \quad 93.7 \%$ $(n=326 / 348)$, and $87.7 \% \quad(n=142 / 162)$ for olaparib-, niraparib-, and rucaparib-treated patients, respectively (Table 2). The risk of any CEI was higher with niraparib than with olaparib (OR 3.36, 95\% CI 2.00-5.65) and with niraparib than with rucaparib (OR 2.09, 95\% CI 1.10-3.95). There was no statistically significant difference in the risk of experiencing any CEI with rucaparib compared with olaparib (OR 1.61, 95\% CI 0.93-2.79; Fig. 1). The percentage of patients who experienced hematologic CEIs during follow-up was $46.2 \%, 64.7 \%$, and $53.7 \%$ for olaparib-, niraparib-, and rucaparib-treated patients, respectively (Table 2 ). The risk of experiencing a hematologic CEI was higher with niraparib than with olaparib (OR 2.13, 95\% CI 1.55-2.92) and with niraparib than with rucaparib (OR 1.58, 95\% CI 1.08-2.30; Fig. 1).

Multivariable models stratified for the presence of a relevant CEI at baseline and adjusted for index PARPi regimen, CCI score, prior treatment with bevacizumab, and cancer-related surgery during the baseline period confirmed that there was a significantly greater probability of any CEI in the follow-up period with niraparib than with olaparib (HR 1.35, 95\% CI 1.14-1.59; $P=0.0005)$; the difference in probability of experiencing any CEI between rucaparib and olaparib (HR 1.16, 95\% CI $0.94-1.43 ; P=0.16)$ and niraparib and rucaparib (HR 1.16, 95\% CI 0.95-1.41; $P=0.15$ ) did not reach statistical significance (Supplementary Table 4 and Supplementary Fig. 3). Median (IQR) time to first CEI was similar between treatment groups at 23.0 (55.0), 24.0 (38.0), and 24.5 (54.0) days for olaparib-, niraparib-, and rucaparib-treated patients, respectively. However, median [IQR] time to first hematologic CEI was longer with olaparib (62.0 [110.5] days) and rucaparib (49.0 [115.0] days) than with niraparib (41.0 [64.0] days).

\section{Dose Modifications}

The proportion of patients initiating treatment with PARPi at the highest indicated dose was $89.4 \% \quad(n=271 / 303) \quad$ for olaparib, $69.3 \%$ $(n=241 / 348)$ for niraparib, and $93.2 \%$ $(n=151 / 162)$ for rucaparib (Table 3$)$. Of those receiving olaparib, $21.1 \%$ required a dose decrease, compared with $35.1 \%(P=0.00009)$ 
Table 1 Baseline demographic and clinical characteristics of patients with ovarian cancer by treatment group

\begin{tabular}{|c|c|c|c|}
\hline & $\begin{array}{l}\text { Olaparib } \\
N=303\end{array}$ & $\begin{array}{l}\text { Niraparib } \\
N=348\end{array}$ & $\begin{array}{l}\text { Rucaparib } \\
N=162\end{array}$ \\
\hline \multicolumn{4}{|l|}{ Demographic characteristics $^{a}$} \\
\hline \multicolumn{4}{|l|}{ Age, years } \\
\hline Mean (SD) & $58.4(10.3)$ & $59.4(9.4)$ & $58.3(8.5)$ \\
\hline Median [IQR] & $59.0[11.0]$ & $59.0[11.0]$ & $58.0[10.0]$ \\
\hline \multicolumn{4}{|l|}{ Age category, $n(\%)$} \\
\hline $18-44$ years & $22(7.3)$ & $17(4.9)$ & $8(4.9)$ \\
\hline $45-64$ years & $224(73.9)$ & $257(73.9)$ & $129(79.6)$ \\
\hline$\geq 65$ years & $57(18.8)$ & $74(21.3)$ & $25(15.4)$ \\
\hline \multicolumn{4}{|l|}{ Payer, $n(\%)$} \\
\hline Commercial & $243(80.2)$ & $271(77.9)$ & $135(83.3)$ \\
\hline Medicare supplemental & $60(19.8)$ & $77(22.1)$ & $27(16.7)$ \\
\hline \multicolumn{4}{|l|}{ Index year, $n(\%)$} \\
\hline 2017 & $82(27.1)$ & $173(49.7)^{* * *}$ & $66(40.7)^{* *}$ \\
\hline 2018 & $126(41.6)$ & $134(38.5)$ & $79(48.8)$ \\
\hline 2019 & $95(31.4)$ & $41(11.8)^{* * *}$ & $17(10.5)^{* * *}$ \\
\hline \multicolumn{4}{|l|}{ Duration of follow-up (days) } \\
\hline Mean (SD) & $278.9(206.9)$ & $341.8(214.7)^{* * *}$ & $318.9(207.3)^{*}$ \\
\hline Median [IQR] & $224.0[293.0]$ & $295.5[339.0]$ & $265.0[275.0]$ \\
\hline Patients with $\geq 90$ days of follow-up, $n(\%)$ & $242(79.9)$ & $307(88.2)^{* *}$ & $145(89.5)^{* *}$ \\
\hline \multicolumn{4}{|l|}{ Clinical characteristics ${ }^{\mathrm{b}}$} \\
\hline \multicolumn{4}{|l|}{ NCI-adapted CCI } \\
\hline Mean (SD) & $0.7(1.0)$ & $0.9(1.1)$ & $0.8(1.1)$ \\
\hline Median [IQR] & $0.0[1.0]$ & $1.0[1.0]$ & $0.5[1.0]$ \\
\hline \multicolumn{4}{|l|}{ Baseline treatment history, $n(\%)$} \\
\hline Systemic chemotherapy & $269(88.8)$ & $315(90.5)$ & $146(90.1)$ \\
\hline Cancer-related surgery & $205(67.7)$ & $250(71.8)$ & $110(67.9)$ \\
\hline Evidence of $B R C A$ testing, $n(\%)$ & $167(55.1)$ & $194(55.7)$ & $97(59.9)$ \\
\hline Evidence of metastatic disease, $n(\%)^{c}$ & $247(81.5)$ & $287(82.5)$ & $135(83.3)$ \\
\hline Regional metastases & $71(23.4)$ & $74(21.3)$ & $29(17.9)$ \\
\hline
\end{tabular}


Table 1 continued

\begin{tabular}{|c|c|c|c|}
\hline & $\begin{array}{l}\text { Olaparib } \\
N=303\end{array}$ & $\begin{array}{l}\text { Niraparib } \\
N=348\end{array}$ & $\begin{array}{l}\text { Rucaparib } \\
N=162\end{array}$ \\
\hline Distant metastases & $176(58.1)$ & $213(61.2)$ & $106(65.4)$ \\
\hline \multicolumn{4}{|c|}{$\begin{array}{l}C C I \text { Charlson Comorbidity Index, } I Q R \text { interquartile range, } N C I \text { National Cancer institute, } S D \text { standard deviation } \\
{ }^{*} P<0.05 \text { versus olaparib; }{ }^{* *} P<0.01 \text { versus olaparib; }{ }^{* * *} P<0.001 \text { versus olaparib; statistical comparisons were performed } \\
\text { using chi-squared or Fisher's exact tests for categorical variables and } t \text { tests for continuous variables } \\
\text { a Demographic characteristics were measured on the date of first PARP inhibitor prescription (index) } \\
{ }^{b} \text { Clinical characteristics were measured during the } 6 \text {-month baseline period excluding baseline treatment history and } \\
\text { evidence of } B R C A \text { testing, which was based on all available patient data in the patient's history }\end{array}$} \\
\hline
\end{tabular}

and $30.2 \%(P=0.029)$ with niraparib and rucaparib, respectively.

Multivariable models stratified by presence of a relevant CEI at baseline and adjusted for a priori confounders during the baseline period confirmed that there was a greater risk of discontinuing treatment with niraparib (HR 1.52, 95\% CI 1.24-1.86) or rucaparib (HR 1.42, 95\% CI 1.11-1.18) than with olaparib (Supplementary Table 5).

Of those patients with at least 6 months' follow-up after PARPi initiation (olaparib $n=172$, niraparib $n=251$, rucaparib $n=117$ ), the proportion persistent with their PARPi (no treatment gaps of more than 90 days) was $62.2 \%$ for olaparib, compared with $35.9 \%$ with niraparib, and $48.7 \%$ with rucaparib $(P<0.001$ niraparib vs olaparib; $P=0.030$ niraparib vs rucaparib; $P=0.023$ rucaparib vs olaparib; Fig. 2). Of patients receiving olaparib, $80.2 \%$ were adherent to the PARPi regimen, as indicated by MPR $\geq 80 \%$, compared with $38.6 \%$ of patients receiving niraparib and $63.2 \%$ of patients receiving rucaparib $(P<0.001$ niraparib vs olaparib, niraparib vs rucaparib, and rucaparib vs olaparib; Fig. 2).

\section{Healthcare Resource Utilization and Cost}

HCRU was similar across treatment groups at baseline, although baseline total medical costs were higher with niraparib $(\$ 92,973)$ compared with olaparib $(\$ 77,595 ; P=0.014)$ but not rucaparib $(\$ 80,042 ; \quad P=0.11 ;$ Supplementary Table 6).
During the follow-up period, $20.1 \%(n=61$ ) 303) of patients receiving olaparib had an inpatient admission, compared with $27.0 \%$ $(n=94 / 348 ; P=0.040)$ receiving niraparib and $30.9 \%(n=50 / 162 ; P=0.010)$ receiving rucaparib (Table 4). A similar pattern was observed for other outpatient services: mean (SD) number of services PPPM was 2.8 (2.1), 3.3 (2.0), and 3.4 (2.2) for patients receiving olaparib, niraparib, and rucaparib, respectively $(P=0.00072$ for niraparib vs olaparib and $P=0.0015$ for rucaparib vs olaparib). Overall, $27.7 \%, 34.8 \%$, and $29.6 \%$ of patients receiving olaparib, niraparib, and rucaparib, respectively, had an ER visit, with no significant difference between the treatment groups. Mean (SD) monthly total medical costs PPPM (excluding pharmacy costs) during follow-up were $\$ 5393$ (\$8828) for olaparib, $\$ 7732(\$ 14,054)$ for niraparib $(P=0.013$ vs olaparib), and $\$ 6868$ (\$7929) for rucaparib $(P=0.076$ vs olaparib).

\section{Sensitivity Analyses}

The proportion of patients experiencing incident CEIs during PARPi treatment by starting dose, specifically below or at the highest indicated dose, was examined. The proportion of patients experiencing an incident CEI in those initiating niraparib below the highest indicated dose was $72.9 \%$, compared with $60.1 \%$ of those initiating olaparib at the highest indicated dose (Supplementary Table 6); the difference in incident CEI was $12.8 \%$ (95\% CI 2.08-22.38; $P<0.05)$. There was no difference $(0.1 \%$ $[95 \% \mathrm{CI}-11.1$ to $10.8 ; P=0.99])$ in the 
Table 2 Prevalence of CEIs during the follow-up period by index PARPi regimen

\begin{tabular}{|c|c|c|c|}
\hline Presence of CEI, $\boldsymbol{n}(\%)$ & $\begin{array}{l}\text { Olaparib } \\
N=303\end{array}$ & $\begin{array}{l}\text { Niraparib } \\
N=348\end{array}$ & $\begin{array}{l}\text { Rucaparib } \\
N=162\end{array}$ \\
\hline Any & $247(81.5)$ & $326(93.7)^{* * *}$ & $142(87.7)^{\dagger}$ \\
\hline Hematologic & $140(46.2)$ & $225(64.7)^{* * *}$ & $87(53.7)^{\dagger}$ \\
\hline AML/MDS & $3(1.0)$ & $1(0.3)$ & $3(1.9)$ \\
\hline AML & $0(0)$ & $1(0.3)$ & $0(0)$ \\
\hline MDS & $3(1.0)$ & $0(0)$ & $3(1.9)$ \\
\hline Anemia $^{a}$ & $118(38.9)$ & $165(47.4)^{*}$ & $68(42.0)$ \\
\hline Leukopenia/neutropenia ${ }^{\mathrm{b}}$ & $51(16.8)$ & $82(23.6)^{*}$ & $37(22.8)$ \\
\hline Thrombocytopenia or transfusion & $56(18.5)$ & $146(42.0)^{* * *}$ & $50(30.9)^{* *, \dagger}$ \\
\hline Other & $231(76.2)$ & $310(89.1)^{* * *}$ & $138(85.2)^{*}$ \\
\hline Acute kidney injury & $26(8.6)$ & $48(13.8)^{*}$ & $29(17.9)^{* *}$ \\
\hline Arthralgia & $86(28.4)$ & $89(25.6)$ & $36(22.2)$ \\
\hline Constipation/diarrhea & $56(18.5)$ & $85(24.4)$ & $46(28.4)^{*}$ \\
\hline Constipation & $40(13.2)$ & $67(19.3)^{*}$ & $31(19.1)$ \\
\hline Diarrhea & $20(6.6)$ & $22(6.3)$ & $18(11.1)$ \\
\hline Dermatitis/rash/photosensitivity & $15(5.0)$ & $12(3.4)$ & $13(8.0)^{\dagger}$ \\
\hline Dermatitis/rash & $14(4.6)$ & $12(3.4)$ & $12(7.4)^{\dagger}$ \\
\hline Photosensitivity & $1(0.3)$ & $0(0)$ & $1(0.6)$ \\
\hline Fatigue & $78(25.7)$ & $99(28.4)$ & $49(30.2)$ \\
\hline Hypertension & $96(31.7)$ & $159(45.7)^{* * *}$ & $65(40.1)$ \\
\hline Infection & $120(39.6)$ & $184(52.9)^{* *}$ & $91(56.2)^{* *}$ \\
\hline Insomnia & $16(5.3)$ & $35(10.1)^{*}$ & $16(9.9)$ \\
\hline Nausea/vomiting & $81(26.7)$ & $118(33.9)^{*}$ & $71(43.8)^{* * *, \dagger}$ \\
\hline Nausea & $80(26.4)$ & $115(33.0)$ & $69(42.9)^{* * *, \dagger}$ \\
\hline Vomiting & $9(3.0)$ & $16(4.6)$ & $12(7.5)^{*}$ \\
\hline Pneumonitis & $0(0)$ & $3(0.9)$ & $2(1.2)$ \\
\hline Transaminitis & $0(0)$ & $4(1.1)$ & $3(1.9)$ \\
\hline
\end{tabular}

$A M L$ acute myeloid leukemia, $C E I$ clinical event of interest, $M D S$ myelodysplastic syndromes, PARPi poly(ADP-ribose) polymerase inhibitors

${ }^{*} P<0.05$ versus olaparib; ${ }^{* *} P<0.01$ versus olaparib; ${ }^{* *} P<0.001$ versus olaparib; ${ }^{\dagger} P<0.05$ versus niraparib; statistical comparisons were performed using chi-squared or Fisher's exact tests for categorical variables and $t$ tests for continuous variables; statistical tests were carried out when there was a frequency of CEIs of at least $1 \%$ per treatment group to avoid comparisons in small sample sizes

${ }^{a}$ CEI based on total claims comprising a diagnosis code only

${ }^{\mathrm{b}} \mathrm{CEI}$ based on total claims comprising a diagnosis code only, a procedure code only, or both a diagnosis and procedure code. Index is date of PARPi initiation 

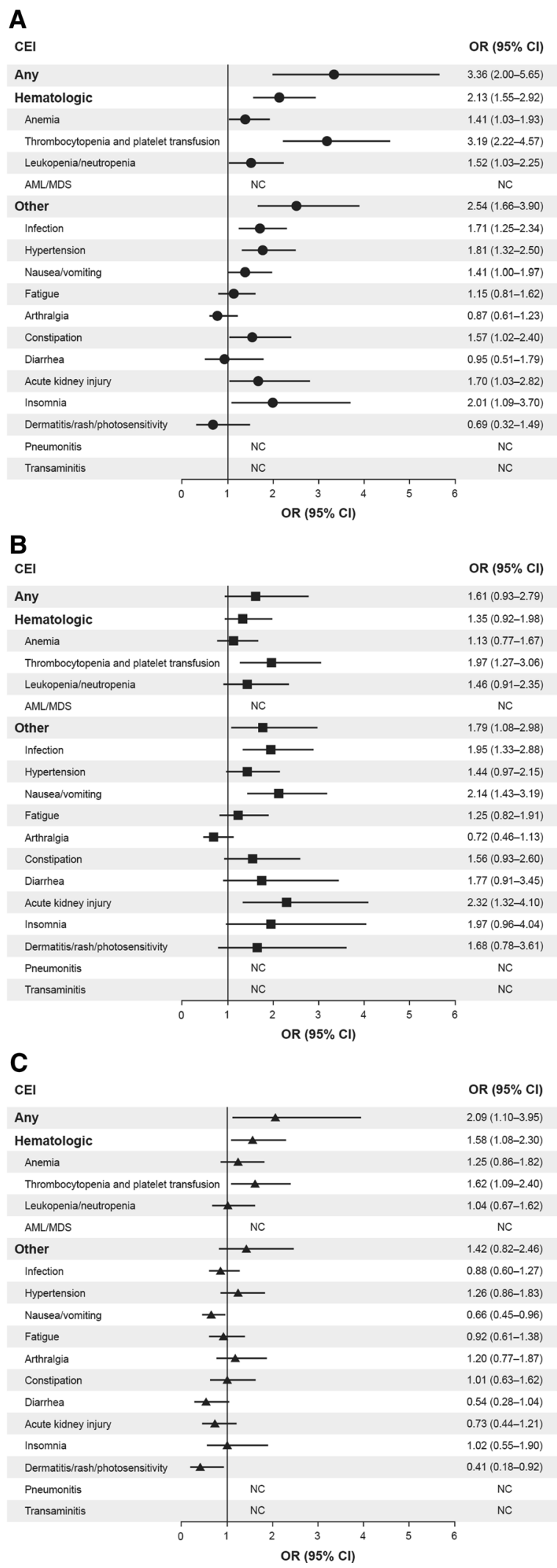

4Fig. 1 Forest plot showing odds ratios and 95\% confidence intervals for comparison of selected clinical events of interest in women with ovarian cancer treated with olaparib, niraparib, or rucaparib as the index PARPi regimen (2017-2019): a niraparib $(N=348) /$ olaparib $(N=303)$; b rucaparib $(N=162)$ /olaparib $(N=303)$; c niraparib $(N=348) /$ rucaparib $(N=162)$. OR $>1$ favors the second drug in the comparison. CEIs listed as NC were not calculated because of the small number of events per cell ( $<3$ patients per group). AML acute myeloid leukemia, CI confidence interval, CEI clinical event of interest, MDS myelodysplastic syndromes, NC not calculable, OR odds ratio, PARPi poly(ADP-ribose) polymerase inhibitors

proportion of patients experiencing an incident CEI in those initiating niraparib below the highest indicated dose $(72.9 \%)$ and at the highest indicated dose (72.8\%; Supplementary Table 7).

Among patients with at least 6 months' continuous enrollment, a reduction in the permitted treatment gap from more than 90 days to more than 45 days did not qualitatively alter the results for persistence (Supplementary Table 8).

\section{DISCUSSION}

This large real-world comparison of PARPi therapy in women with OC leveraged US healthcare claims data and demonstrated key differences between the three FDA-approved PARPi in the risk of experiencing a CEI, likelihood of dose modifications, ability to receive continuous PARPi therapy, and HCRU and total medical costs. Demographics, clinical characteristics, and CEIs were similar between groups in this large cohort at baseline, while the approved indications during the index period (January 1, 2017, to May 31, 2019) meant that the majority of patients were likely to be receiving maintenance therapy. Indeed, approximately $90 \%$ of patients across all groups had a record of systemic chemotherapy in their treatment history at baseline.

CEIs observed during the index period were consistent with the safety endpoints from pivotal RCTs with olaparib, niraparib, and 
Table 3 Dose modifications and persistence throughout the index PARPi regimen

\begin{tabular}{|c|c|c|c|}
\hline & $\begin{array}{l}\text { Olaparib } \\
N=303\end{array}$ & $\begin{array}{l}\text { Niraparib } \\
N=348\end{array}$ & $\begin{array}{l}\text { Rucaparib } \\
N=162\end{array}$ \\
\hline \multicolumn{4}{|l|}{ Treatment duration of index PARPi regimen among all patients, $n(\%)$} \\
\hline$<90$ days & $215(71.0)$ & $209(60.1)^{* *}$ & $95(58.6)^{* * *}$ \\
\hline$\geq 90$ days & $88(29.0)$ & $139(39.9)^{* *}$ & $67(41.4)^{* * *}$ \\
\hline Mean initial daily dose ${ }^{\mathrm{a}}, \mathrm{mg} /$ day $(\mathrm{SD})$ & $597.4(108.2)$ & $265.2(55.6)$ & $1242.0(669.3)$ \\
\hline \multicolumn{4}{|l|}{ Initial PARPi dose category, $n(\%)$} \\
\hline Highest indicated dose ${ }^{b}$ & $271(89.4)$ & $241(69.3)$ & $151(93.2)$ \\
\hline \multicolumn{4}{|l|}{ PARPi daily dose ${ }^{\mathrm{a}}$ change from initial dose, $n(\%)$} \\
\hline No dosage change & $261(86.1)$ & $233(67.0)^{* * *}$ & $121(74.7)^{* *}$ \\
\hline Dosage decrease & $64(21.1)$ & $122(35.1)^{* * *}$ & $49(30.2)^{*}$ \\
\hline Dosage increase & $10(3.3)$ & $24(6.9)^{*}$ & $5(3.1)$ \\
\hline Patients with at least 6 months' continuous enrollment, $n(\%)$ & $172(56.8)$ & $251(72.1)^{* * *}$ & $117(72.2)^{* *}$ \\
\hline \multicolumn{4}{|l|}{ Time to non-persistence ${ }^{c}$, days } \\
\hline Mean (SD) & $97.8(46.1)$ & $92.2(50.0)$ & $94.2(49.5)$ \\
\hline Median $[\mathrm{IQR}]$ & $89.5[78.0]$ & $89.0[77.0]$ & $89.0[73.0]$ \\
\hline \multicolumn{4}{|l|}{$\mathrm{MPR}^{\mathrm{d}}$} \\
\hline Mean (SD) & $0.7(0.3)$ & $0.6(0.3)^{* * *}$ & $0.7(0.3)^{\dagger \dagger \dagger}$ \\
\hline Median [IQR] & $0.7[0.7]$ & $0.5[0.5]$ & $0.7[0.7]$ \\
\hline
\end{tabular}

$I Q R$ interquartile range, $M P R$ medication possession ratio, $P A R P i$ poly(ADP-ribose) polymerase inhibitors, $S D$ standard deviation

${ }^{*} P<0.05$ versus olaparib; ${ }^{* *} P<0.01$ versus olaparib; ${ }^{* * *} P<0.001$ versus olaparib; ${ }^{\dagger \dagger \dagger} P<0.001$ versus niraparib; statistical comparisons were performed using chi-squared or Fisher's exact tests for categorical variables and $t$ tests for continuous variables. Statistical comparisons were not performed for the mean initial daily dose or the initial PARPi dose category

${ }^{a}$ Daily dose was calculated as (quantity supplied $\times$ drug strength)/days' supply. Initial daily dose was examined at the first claim only

${ }^{b}$ Highest indicated doses were taken from the prescribing information for each PARPi as 600, 800, 300, and 1200 mg/day for olaparib tablets, olaparib capsules, niraparib capsules, and rucaparib tablets, respectively

${ }^{c}$ Days to non-persistence were determined among the subset of patients who had at least 6 months of follow-up; persistence was indicated by no treatment gaps of more than 90 days during the 6-month post-index period [21]

${ }^{\mathrm{d}}$ MPR was measured as the ratio of the sum of days' supply during the fixed 6-month (180-day) follow-up period among the subset of patients with at least 6 months of follow-up [21]

rucaparib $[7,8,13-17,30,31]$ and the limited real-world evidence published to date [19-21, 23]. The most common CEIs observed included nausea, fatigue, vomiting, and hematologic toxicities such as anemia and thrombocytopenia. Hematologic toxicities are a common class effect of PARPi, often occurring early after treatment initiation; they are also the most common cause of dose modification, interruption, and discontinuation [4]. In our 


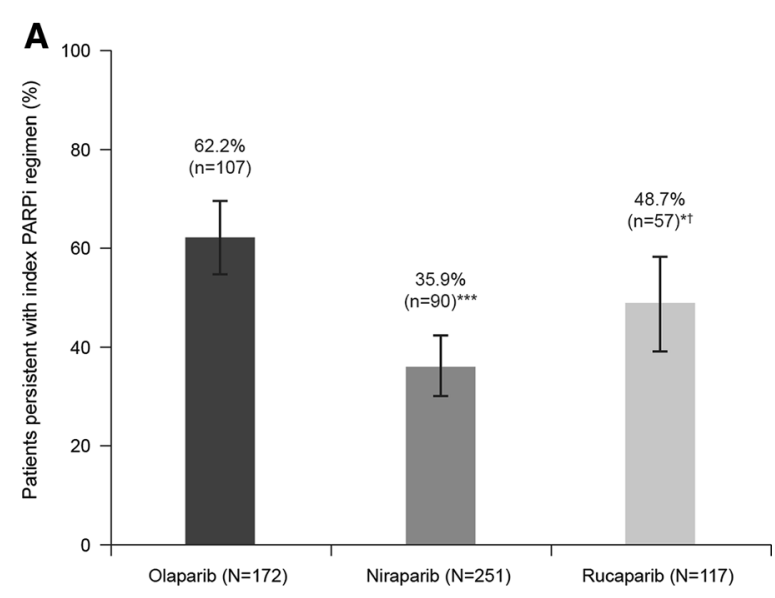

B

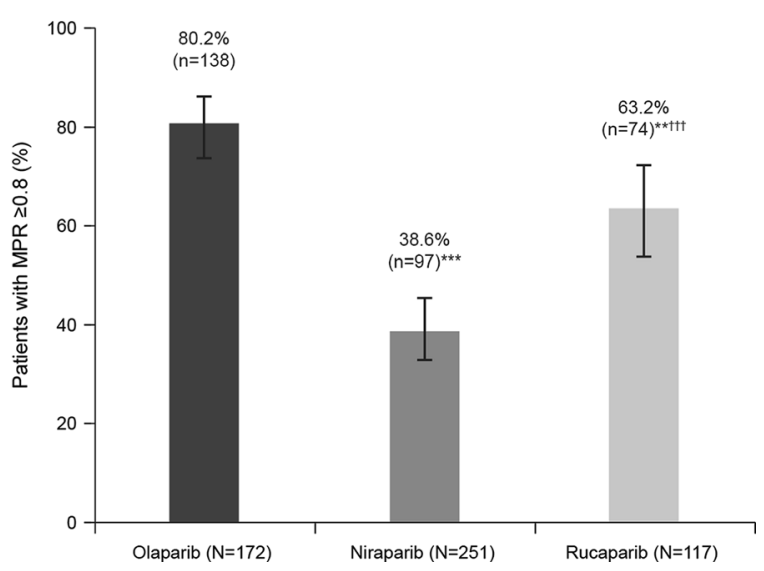

Fig. 2 a Persistence ${ }^{a}$ and $\mathbf{b}$ adherence ${ }^{b}$ to index PARPi regimen. ${ }^{*} P<0.05$ versus olaparib; ${ }^{* *} P<0.01$ versus olaparib; ${ }^{* * *} P<0.001$ versus olaparib; ${ }^{\dagger} P<0.05$ versus niraparib; ${ }^{\dagger \dagger} P<0.001$ versus niraparib; statistical comparisons were performed using chi-squared or Fisher's exact tests for categorical variables and $t$ tests for continuous variables. Error bars represent 95\% Clopper-Pearson confidence interval. ${ }^{a}$ Persistence: percentage of patients with no index PARPi regimen treatment gaps of more than 90 days or those who had at least 6 months of continuous enrollment [28]; ${ }^{\mathrm{b}}$ Adherence: MPR was measured as the ratio of the sum of days' supply during the fixed 6-month (180-day) follow-up period, and patients with MPR $<80 \%$ were categorized as nonadherent [28]. MPR medication possession ratio, PARPi poly(ADP-ribose)polymerase inhibitors

study, rates of hematologic CEIs were consistent with RCT data $[7,8]$ and were higher with niraparib, followed by rucaparib and then olaparib. Differences between PARPi in terms of tolerability and dose modifications in pivotal RCTs in the OC maintenance setting have recently been highlighted in an indirect treatment comparison [18]. Specifically, any grade 3-4 AEs were observed less frequently with olaparib than with niraparib and rucaparib [18], which is consistent with the results of a recent network meta-analysis of RCTs [7].

The differences observed in non-hematologic CEIs, including a higher prevalence of insomnia and hypertension with niraparib vs olaparib and of nausea/vomiting and constipation/diarrhea with rucaparib vs olaparib, build on similar results presented in a recent singlecenter analysis of patients with relapsed platinum-sensitive OC treated with PARPi [25]. Offtarget binding to dopamine, serotonin, and norepinephrine receptors has been reported for niraparib and rucaparib $[4,6,11]$ and may explain the differences in the incidence of AEs such as hypertension and insomnia; however, further investigation is required. Abnormalities in liver function tests are commonly observed with rucaparib but are not thought to be related to liver toxicity [32]. Future studies investigating whether any patient characteristics are associated with favorable tolerability profiles of the different PARPi regimens may be interesting to explore.

More dose modifications were observed with niraparib and rucaparib vs olaparib in our study, confirming similar findings in a recent indirect treatment comparison of key RCTs in the maintenance OC setting [18]. The most frequent AEs experienced with PARPi (regardless of PARPi prescribed), such as nausea, fatigue, anemia, and vomiting, are usually transient, manageable (grade 1), and unlikely to lead to drug discontinuation if supportive care is provided $[12,33]$. Dose reductions and interruptions may be required for more severe events (grade 2 or worse), such as thrombocytopenia, and discontinuation is necessary for severe events that cannot otherwise be managed (grade 3 or 4 lasting more than 28 days at the lowest PARPi dose). Each PARPi has a recommended schedule of dose modifications that allows for continued use [9-11]. Niraparib dose modifications may be linked to protocol-mandated changes in the 
Table 4 All-cause healthcare resource utilization and costs PPPM measured during the follow-up period by index PARPi regimen

\begin{tabular}{|c|c|c|c|}
\hline & $\begin{array}{l}\text { Olaparib } \\
N=303\end{array}$ & $\begin{array}{l}\text { Niraparib } \\
N=348\end{array}$ & $\begin{array}{l}\text { Rucaparib } \\
N=162\end{array}$ \\
\hline \multicolumn{4}{|l|}{ Inpatients } \\
\hline Patients with an admission, $n(\%)$ & $61(20.1)$ & $94(27.0)^{*}$ & $50(30.9)^{*}$ \\
\hline \multicolumn{4}{|l|}{ Number of inpatient admissions } \\
\hline Mean (SD) & $0.0(0.1)$ & $0.1(0.2)$ & $0.1(0.1)^{*}$ \\
\hline Median [IQR] & $0.0[0.0]$ & $0.0[0.0]$ & $0.0[0.1]$ \\
\hline \multicolumn{4}{|l|}{ Length of stay, days } \\
\hline Mean (SD) & $1.3(3.4)$ & $2.0(4.5)^{*}$ & $2.1(4.3)^{*}$ \\
\hline Median [IQR] & $0.0[0.0]$ & $0.0[2.0]$ & $0.0[3.0]$ \\
\hline \multicolumn{4}{|l|}{ Outpatient ER visits } \\
\hline Patients with an ER visit, $n$ (\%) & $84(27.7)$ & $121(34.8)$ & $48(29.6)$ \\
\hline \multicolumn{4}{|l|}{ Number of ER visits } \\
\hline Mean (SD) & $0.1(0.2)$ & $0.1(0.2)$ & $0.1(0.1)$ \\
\hline Median [IQR] & $0.0[0.1]$ & $0.0[0.1]$ & $0.0[0.1]$ \\
\hline \multicolumn{4}{|l|}{ Outpatient office visits } \\
\hline Patients with an office visit, $n(\%)$ & $296(97.7)$ & $342(98.3)$ & $158(97.5)$ \\
\hline \multicolumn{4}{|l|}{ Number of office visits } \\
\hline Mean (SD) & $1.4(0.8)$ & $1.7(1.0)^{* *}$ & $1.5(0.7)^{\dagger}$ \\
\hline Median [IQR] & $1.3[0.9]$ & $1.5[1.2]$ & $1.4[0.7]$ \\
\hline \multicolumn{4}{|l|}{ Other outpatient services } \\
\hline Patients with another outpatient service, $n(\%)$ & $301(99.3)$ & $348(100.0)^{* * *}$ & $160(98.8)^{\dagger}$ \\
\hline \multicolumn{4}{|l|}{ Number of services } \\
\hline Mean (SD) & $2.8(2.1)$ & $3.3(2.0)^{* *}$ & $3.4(2.2)^{* *}$ \\
\hline Median [IQR] & $2.1[1.8]$ & $2.9[2.2]$ & $2.8[2.6]$ \\
\hline \multicolumn{4}{|l|}{ Outpatient pharmacy } \\
\hline Patients with a prescription, $n(\%)$ & $303(100.0)$ & $348(100.0)^{\mathrm{b}}$ & $162(100.0)^{\mathrm{b}}$ \\
\hline \multicolumn{4}{|l|}{ Number of prescriptions } \\
\hline Mean (SD) & $3.3(2.2)$ & $3.5(2.3)$ & $3.3(1.9)$ \\
\hline Median [IQR] & $2.7[2.2]$ & $3.0[2.4]$ & $3.0[2.2]$ \\
\hline Mean monthly inpatient costs $(\mathrm{SD})^{\mathrm{a}}$ & $\$ 1354(\$ 5717)$ & $\$ 2274(\$ 10,265)$ & $\$ 2045(\$ 5516)$ \\
\hline Mean monthly outpatient costs $(\mathrm{SD})^{\mathrm{a}}$ & $\$ 4038(\$ 5926)$ & $\$ 5459(\$ 7469)^{* *}$ & $\$ 4822(\$ 5237)$ \\
\hline ER visits & $\$ 146(\$ 456)$ & $\$ 161(\$ 480)$ & $\$ 105(\$ 287)$ \\
\hline
\end{tabular}


Table 4 continued

\begin{tabular}{llll}
\hline & $\begin{array}{l}\text { Olaparib } \\
\boldsymbol{N}=\mathbf{3 0 3}\end{array}$ & $\begin{array}{l}\text { Niraparib } \\
\boldsymbol{N}=\mathbf{3 4 8}\end{array}$ & $\begin{array}{l}\text { Rucaparib } \\
\boldsymbol{N}=\mathbf{1 6 2}\end{array}$ \\
\hline Outpatient office visits & $\$ 248(\$ 193)$ & $\$ 277(\$ 239)$ & $\$ 261(\$ 228)$ \\
Other outpatient services & $\$ 3644(\$ 5748)$ & $\$ 5021(\$ 7304)^{* *}$ & $\$ 4456(\$ 5103)$ \\
Mean monthly total medical costs, excluding pharmacy $(\mathrm{SD})^{\mathrm{a}}$ & $\$ 5393(\$ 8828)$ & $\$ 7732(\$ 14,054)^{*}$ & $\$ 6868(\$ 7929)$ \\
Mean monthly total costs $(\mathrm{SD})^{\mathrm{a}}$ & $\$ 16,768(\$ 8705)$ & $\$ 16,737(\$ 13,981)$ & $\$ 17,885(\$ 8139)$ \\
\hline
\end{tabular}

$E R$ emergency room, $I Q R$ interquartile range, $P A R P i$ poly(ADP-ribose) polymerase inhibitors, PPPM per patient per month, $S D$ standard deviation

${ }^{*} P<0.05$ versus olaparib; ${ }^{* *} P<0.01$ versus olaparib; ${ }^{* * *} P<0.001$ versus olaparib; ${ }^{\dagger} P<0.05$ versus niraparib; statistical comparisons were performed using chi-squared or Fisher's exact tests for categorical variables and $t$ tests for continuous variables

${ }^{a}$ All dollar estimates are reported PPPM to account for variation in duration of the index PARPi regimen and were adjusted for inflation using the Medical Care Component of the Consumer Price Index and standardized to the US dollar value in 2018. In addition to costs, rows representing number of services have also been standardized to PPPM

${ }^{\mathrm{b}}$ Not calculable

recommended dosing regimen for patients with specific toxicities in the ENGOT-OV16/NOVA study. A subsequent post hoc analysis reported that patients with a weight of less than $77 \mathrm{~kg}$ or a baseline platelet count of less than 150,000/ $\mathrm{mm}^{3}$ benefited from a reduced starting dose of $200 \mathrm{mg}$ once daily [34], which led to a label update (April 2020) recommending dosing according to body weight and platelet levels [11]. In our study, fewer patients receiving niraparib $(69.3 \%)$ than olaparib (89.4\%) or rucaparib (93.2\%) started treatment at the highest indicated dose, suggesting that modified niraparib starting doses were implemented in routine practice to mitigate the risk of severe hematologic toxicity. In a sensitivity analysis, we found the rate of hematologic toxicities in patients starting at a lower niraparib dose remained significantly higher than the rate in patients receiving olaparib or rucaparib at any starting dose. This observation is consistent with the prospective exploratory analysis of the first-line PRIMA study, which showed a lower but persistent high rate of thrombocytopenia ( $21 \%$ grade 3 or worse) in patients receiving the modified dosing regimen based on baseline body weight and platelet count [11]. Whether changes in the recommended dosing regimen for niraparib [14] affected its tolerability and persistence requires further investigation.
Non-adherence to recommended dosing regimens can contribute greatly to the variability observed in a drug's therapeutic effect and can also affect clinical outcome $[35,36]$. In a recent retrospective cohort study of women with OC, a quarter of patients were suboptimally adherent to PARPi therapy and non-adherent patients were more likely to be receiving niraparib and have a shorter duration of therapy [37]. In our study, the ability to receive continuous PARPi therapy also differed between the PARPi, with lower persistence observed with niraparib and rucaparib vs olaparib. Previous studies have shown fewer treatment interruptions [18] and longer duration of use [23] with olaparib compared with niraparib and rucaparib. In a sensitivity analysis including persistent patients only (i.e., those with no treatment gaps of more than 90 days in the 6 months after starting PARPi), we found no difference in the association between rate of CEI and index PARPi regimen, suggesting that differences in rates of CEIs between the PARPi may be driven by nonpersistent patients; however, as reason for discontinuation is not available in claims data, we could not explicitly determine whether discontinuation of treatment was due to the CEI. It is also unclear if discontinuation was due to disease progression or any associated changes in therapy. 
Whether a decrease in PARPi persistence or reductions in dose were translated into longerterm reductions in effectiveness remains an important focus for future research. Nevertheless, initial follow-up data from PARPi clinical trials in newly diagnosed and relapsed patients with OC indicated good long-term tolerability and efficacy [38, 39]. Indeed, long-term followup of olaparib RCTs demonstrated that many patients with platinum-sensitive relapsed OC remain on therapy for several years; $13 \%$ and $22 \%$ of patients remained on olaparib at 5 years in Study 19 and SOLO2 (BRCA mutated), respectively $[39,40]$. Tolerability is likely to be an important factor for long-term PARPi maintenance therapy, and further follow-up data in this setting are awaited.

In addition to effectiveness and tolerability, the HCRU and costs associated with PARPi therapy aid understanding of the full patient experience. Here, inpatient admissions and outpatient service use were higher for niraparib and rucaparib than for olaparib, reflected in their respective total medical costs (excluding pharmacy costs). Higher medical costs with niraparib vs olaparib and rucaparib were previously reported in a US real-world study in patients with OC undergoing PARPi treatment following first-line platinum-based therapy [41]. Patients receiving niraparib need to be monitored weekly for the first month of treatment, which may increase medical costs and place an extra travel burden on patients.

Baseline total medical costs were higher in the niraparib group than in the olaparib and rucaparib groups, which was driven by higher costs for outpatient services. Inpatient, ER, and office visit costs were not higher. This could partly be explained by the approval of niraparib solely for maintenance treatment of patients with a complete or partial response to secondline platinum-based chemotherapy during the study period. Thus, the high costs were likely driven by other outpatient services related to chemotherapy infusion during the baseline assessment.

To our knowledge, this study is the largest real-world comparison of PARPi therapy in women with OC. While we cannot discount the possibility that some patients in our study received PARPi in a treatment setting, our index period focused on a time when all three PARPi were approved for maintenance use. The smaller number of patients who received rucaparib $(n=162)$ in this window, compared with olaparib $(n=303)$ and niraparib $(n=348)$, may be explained by the April 2018 extension of the rucaparib label to include second-line maintenance therapy in platinum-sensitive patients [11]; the majority of patients who initiated rucaparib in this study did so in 2018. A greater proportion of olaparib-prescribed patients initiated treatment in 2019 compared with niraparib or rucaparib, resulting in less time for follow-up before the May 31, 2019 cutoff. Consequently, the duration of follow-up and proportion of patients with 6 months' continuous follow-up differed across the individual PARPi and appeared shorter in the olaparib cohort, although this does not affect interpretation of adherence and persistence data.

Use of claims data is well established in research examining tolerability and HCRU in several diseases, including a range of cancers [42-45]. Nevertheless, this study has some limitations, including biases inherent to observational studies and use of healthcare claims data, which are recorded for billing rather than research purposes and do not include a review of medical records. The incidence of CEIs was generally lower in our analysis vs clinical trials; only CEIs that were severe enough to seek medical attention or that needed treatment were recorded, but this is unlikely to be differential with respect to the PARPi. While common toxicities such as nausea and fatigue may not be recorded in claims data unless severe, they are still problematic for patients and an important consideration for treating physicians. Rates of hypertension and infection were generally higher in our study than in clinical trials, perhaps because of the wide range of terms captured to compile these CEIs, but this may also be more representative of real-world experience. The dosing, adherence, and persistence results reflect PARPi prescription patterns and may not fully capture usage. Line of therapy was not recorded in claims, and we could not with certainty assign PARPi use as maintenance or treatment. Certain variables, such as reasons for 
PARPi discontinuation, OC stage, and BRCA status, were not captured. Some patients may have switched insurers before the study inclusion period, rendering their full history inaccessible, which may explain why approximately $10 \%$ of patients had no systemic chemotherapy prescription at baseline. Multiple statistical tests were carried out and we did not account for multiplicity of testing, increasing the probability of a statistically significant finding being observed by chance. Although some comparisons were adjusted for potential a priori confounders, residual confounding factors may have biased the study estimates. However, results from our primary analytical cohort were supported by the sensitivity analyses conducted. Finally, this study only included patients in the USA, but the results are generalizable to the wider population of commercially insured patients with OC. Similar studies are planned in other countries.

\section{CONCLUSION}

This is the largest real-world comparison of PARPi therapy in women with OC conducted to date. The results suggest differences in the risk of experiencing a CEI, the likelihood of dose modifications, the ability to receive continuous PARPi therapy, HCRU, and total medical costs between the PARPi. This study will help inform physicians and payers of the importance of considering the respective tolerability profiles of PARPi, plus the potential downstream implications for treatment adherence/persistence and health economics, when selecting the most appropriate maintenance therapy for patients with OC. Further research will assess the influence of medication adherence on long-term effectiveness of PARPi using patient records.

\section{ACKNOWLEDGMENTS}

Funding. This study was funded by AstraZeneca and is part of an alliance between AstraZeneca and Merck Sharp \& Dohme Corp, a subsidiary of Merck \& Co, Inc, Kenilworth, NJ,
USA. AstraZeneca also funded the rapid service and open-access fees.

Medical Writing, Editorial Assistance, and Other Assistance. Under the direction of the authors, writing and editorial support in the preparation of this manuscript was provided by Carol Wareham, MRes, PhD and Ben Drever, PhD of AMICULUM Ltd (Bollington, UK), funded by AstraZeneca. We thank Elizabeth Packnet, Nicole M. Zimmerman, Matthew Brouillette, Robert Sedgley, and Deb E. Irwin, PhD of IBM Watson Health ${ }^{\mathrm{TM}}$ for analytical support, funded by AstraZeneca. We also thank Will Sawyer and Miguel Miranda (AstraZeneca) for statistical support.

Authorship. All named authors meet the International Committee of Medical Journal Editors (ICMJE) criteria for authorship for this article, take responsibility for the integrity of the work as a whole, and have given their approval for this version to be published.

Author Contributions. R. C. Arend: Conceptualization; investigation; methodology; writing-original draft, review and editing. D. M. O'Malley: conceptualization; investigation; methodology; writing-original draft, review and editing. S. Banerjee: conceptualization; investigation; methodology; writingoriginal draft, review and editing. K. McLaurin: conceptualization; data curation; formal analysis; investigation; methodology; project administration; validation; visualization; writing-original draft, review and editing. R. Davidson: conceptualization; data curation; formal analysis; investigation; methodology; project administration; validation; visualization; writing-original draft, review and editing. G. H. Long: conceptualization; data curation; formal analysis; investigation; methodology; project administration; validation; visualization; writing-original draft, review and editing.

Prior Presentation. Initial data from this study were presented at the Society of Gynecologic Oncology (SGO) Annual Meeting on Women's Cancer on March 19-25, 2021. 
Disclosures. Rebecca C. Arend reports honoraria for advisory boards from Clovis, Leap Therapeutics, AstraZeneca, Tesaro/GSK, Merck, and Caris Life Sciences and honoraria for steering committees from VBL Therapeutics. David M. O'Malley reports personal fees (consultant/ advisory board) and institutional support for clinical research from AstraZeneca, Clovis, Tesaro/GSK, Immunogen, Janssen/Johnson \& Johnson, AbbVie, Regeneron, Genentech/ Roche, GOG Foundation, Iovance Biotherapeutics, Inc, Eisai, Agenus, Merck, and Mersana, personal fees (consultant/advisory board) from Ambry, Myriad Genetics, Tarveda, Elevar, Novartis, and SeaGen, personal fees (steering committee) and institutional support for clinical research from Amgen, personal fees (steering committee/consultant/advisory board) and institutional support for clinical research from Novocure, personal fees (data and safety monitoring board) from Watermark/TapImmune, and institutional support for clinical research from VentiRx, Array Biopharma, EMD Serono, Ergomed, Ajinomoto, Inc, Ludwig Cancer Research, Stemcentrx, Inc, Cerulean Pharma, Bristol-Myers Squibb Co, Serono, Inc, TRACON Pharmaceuticals, Yale University, New Mexico Cancer Care Alliance, INC Research, Inc, inVentiv Health Clinical, and PRA International. Susana Banerjee reports institutional research funding from AstraZeneca, Tesaro, and GSK, honoraria for advisory boards from AstraZeneca, Amgen, MSD, Clovis, Genmab, Immunogen, Mersana, Oncxerna, Merck Serono, Pfizer, and Roche, honoraria for lectures from AstraZeneca, GSK, Amgen, and Pfizer, and non-compensated involvement in a clinical advisory board for Epsilogen. Gráinne H. Long, Kimmie McLaurin, and Richard Davidson are employees of and own stock in AstraZeneca.

Compliance with Ethics Guidelines. We conducted a retrospective cohort study using the US MarketScan ${ }^{\circledR}$ Commercial and Medicare Supplemental Databases, commercial health insurance claims databases for beneficiaries in the USA. The databases are fully compliant with the Health Insurance Portability and Accountability Act. As the patient and provider data included in these analyses were fully de- identified, this study was exempt from institutional review board approval.

Data Availability. The datasets generated and/or analyzed during the current study are not publicly available. They are the property of MarketScan ${ }^{\circledR}$ and have been licensed by AstraZeneca for this study.

Open Access. This article is licensed under a Creative Commons Attribution-NonCommercial 4.0 International License, which permits any non-commercial use, sharing, adaptation, distribution and reproduction in any medium or format, as long as you give appropriate credit to the original author(s) and the source, provide a link to the Creative Commons licence, and indicate if changes were made. The images or other third party material in this article are included in the article's Creative Commons licence, unless indicated otherwise in a credit line to the material. If material is not included in the article's Creative Commons licence and your intended use is not permitted by statutory regulation or exceeds the permitted use, you will need to obtain permission directly from the copyright holder. To view a copy of this licence, visit http:// creativecommons.org/licenses/by-nc/4.0/.

\section{REFERENCES}

1. Vanacker H, Harter P, Labidi-Galy SI, et al. PARPinhibitors in epithelial ovarian cancer: actual positioning and future expectations. Cancer Treat Rev. 2021;99: 102255.

2. Tomao F, Bardhi E, Di Pinto A, et al. Parp inhibitors as maintenance treatment in platinum sensitive recurrent ovarian cancer: an updated meta-analysis of randomized clinical trials according to BRCA mutational status. Cancer Treat Rev. 2019;80: 101909.

3. Pilié PG, Gay CM, Byers LA, O'Connor MJ, Yap TA. PARP inhibitors: extending benefit beyond BRCAmutant cancers. Clin Cancer Res. 2019;25:3759-71.

4. LaFargue CJ, Dal Molin GZ, Sood AK, Coleman RL. Exploring and comparing adverse events between PARP inhibitors. Lancet Oncol. 2019;20:e15-28. 
5. Pommier $\mathrm{Y}, \mathrm{O}^{\prime}$ Connor $\mathrm{MJ}$, de Bono J. Laying a trap to kill cancer cells: PARP inhibitors and their mechanisms of action. Sci Transl Med. 2016;8: 362 ps17.

6. Leo E, Johannes J, Illuzzi G, et al. A head-to-head comparison of the properties of five clinical PARP inhibitors identifies new insights that can explain both the observed clinical efficacy and safety profiles. Cancer Res. 2018;78(13 Suppl): abst LB-73.

7. Bao S, Yue Y, Hua Y, et al. Safety profile of poly (ADP-ribose) polymerase (PARP) inhibitors in cancer: a network meta-analysis of randomized controlled trials. Ann Transl Med. 2021;9:1229.

8. Hao J, Liu Y, Zhang T, et al. Efficacy and safety of PARP inhibitors in the treatment of advanced ovarian cancer: an updated systematic review and meta-analysis of randomized controlled trials. Crit Rev Oncol Hematol. 2021;157: 103145.

9. AstraZeneca. Lynparza ${ }^{\circledR}$ (olaparib) tablets, for oral use. US prescribing information (2019).

10. Clovis Oncology. Rubraca ${ }^{\circledR}$ (rucaparib) tablets, for oral use. US prescribing information (2020).

11. Tesaro. Zejula (niraparib) capsules, for oral use. US prescribing information (2020).

12. Moore KN, Pothuri B, Monk B, Coleman RL. PARP inhibition in recurrent ovarian cancer. Clin Adv Hematol Oncol. 2020;18:647-55.

13. Moore K, Colombo N, Scambia G, et al. Maintenance olaparib in patients with newly diagnosed advanced ovarian cancer. N Engl J Med. 2018;379: 2495-505.

14. González-Martín A, Pothuri B, Vergote I, et al. Niraparib in patients with newly diagnosed advanced ovarian cancer. N Engl J Med. 2019;381: 2391-402.

15. Mirza MR, Monk BJ, Herrstedt J, et al. Niraparib maintenance therapy in platinum-sensitive, recurrent ovarian cancer. N Engl J Med. 2016;375: 2154-64.

16. Coleman RL, Oza AM, Lorusso D, et al. Rucaparib maintenance treatment for recurrent ovarian carcinoma after response to platinum therapy (ARIEL3): a randomised, double-blind, placebocontrolled, phase 3 trial. Lancet. 2017;390:1949-61.

17. Pujade-Lauraine E, Ledermann JA, Selle F, et al. Olaparib tablets as maintenance therapy in patients with platinum-sensitive, relapsed ovarian cancer and a BRCA1/2 mutation (SOLO2/ENGOT-Ov21): a double-blind, randomised, placebo-controlled, phase 3 trial. Lancet Oncol. 2017;18:1274-84.
18. Sackeyfio A, Nussey F, Friedlander M, Pujade-Lauraine E. Comparative efficacy and tolerability of the PARP inhibitors, olaparib $300 \mathrm{mg}$ tablets BID, niraparib $300 \mathrm{mg}$ capsules QD and rucaparib 600 mg tablets BID as maintenance treatment in BRCAmutated (BRCAm) platinum-sensitive relapsed ovarian. Gynecol Oncol. 2018;149:43-4.

19. Cecere SC, Giannone G, Salutari V, et al. Olaparib as maintenance therapy in patients with BRCA 1-2 mutated recurrent platinum sensitive ovarian cancer: real world data and post progression outcome. Gynecol Oncol. 2020;156:38-44.

20. Marme F, Hilpert F, Welslau M, et al. Olaparib in German routine clinical practice: updated interim results of the non-interventional study C-PATROL. J Clin Oncol. 2018;36(15 Suppl):abst e17549.

21. Marmé F, Hilpert F, Welslau M, et al. Results of the second interim analysis of C-PATROL: a non-interventional study on olaparib within German routine clinical practice. Gynecol Oncol. 2019;154:250-1.

22. Borrelli EP, McGladrigan CG. Real-world evidence of poly ADP-ribose polymerase inhibitors in the treatment of ovarian cancer: a systematic literature review. J Oncol Pharm Pract. 2020;26:1977-86.

23. Eakin CM, Ewongwo A, Pendleton L, Monk BJ, Chase DM. Real world experience of poly (ADP-ribose) polymerase inhibitor use in a community oncology practice. Gynecol Oncol. 2020;159:112-7.

24. Orbegoso Aguilar CMA, Dumas L, Davies E, Gore M, George A, Banerjee S. Olaparib maintenance treatment, the Royal Marsden experience. Ann Oncol. 2018;29(Suppl 9):ix84 (abst 273P).

25. Nagy B, Morgan RD, Jayson GC, Mitchell C, Clamp AR, Hasan JH. Comparison of toxicities of PARP inhibitors used in gynaecological cancers observed at a large cancer centre. Ann Oncol. 2020;31(Suppl 4):S621 (abst 822P).

26. Cramer JA, Roy A, Burrell A, et al. Medication compliance and persistence: terminology and definitions. Value Health. 2008;11:44-7.

27. Adamson DM, Chang S, Hansen L. Health research data for the real world: the MarketScan databases. Stamford: Thomson; 2005.

28. Karve S, Cleves MA, Helm M, Hudson TJ, West DS, Martin BC. Good and poor adherence: optimal cutpoint for adherence measures using administrative claims data. Curr Med Res Opin. 2009;25:2303-10.

29. Strandberg E, Lin X, Ronghui X. Estimation of main effect when covariates have non-proportional hazards. Comm Stat Simul C. 2014;43:1760-70. 
30. Ledermann J, Harter P, Gourley C, et al. Olaparib maintenance therapy in patients with platinumsensitive relapsed serous ovarian cancer: a preplanned retrospective analysis of outcomes by BRCA status in a randomised phase 2 trial. Lancet Oncol. 2014;15:852-61.

31. Swisher EM, Lin KK, Oza AM, et al. Rucaparib in relapsed, platinum-sensitive high-grade ovarian carcinoma (ARIEL2 Part 1): an international, multicentre, open-label, phase 2 trial. Lancet Oncol. 2017;18:75-87.

32. Ledermann JA, Coleman RL. ARIEL3 Investigators clarify the effects of rucaparib on the liver. https:// ascopost.com/issues/november-10-2017/ariel3investigators-clarify-the-effects-of-rucaparib-onthe-liver/. Accessed April 2021.

33. Banerjee S, Gonzalez-Martin A, Harter P, et al. Firstline PARP inhibitors in ovarian cancer: summary of an ESMO Open-cancer horizons round-table discussion. ESMO Open. 2020;5: e001110.

34. Berek JS, Matulonis UA, Peen U, et al. Safety and dose modification for patients receiving niraparib. Ann Oncol. 2018;29:1784-92.

35. Partridge AH, Avorn J, Wang PS, Winer EP. Adherence to therapy with oral antineoplastic agents. J Natl Cancer Inst. 2002;94:652-61.

36. Morgan L. Adherence to ibrutinib therapy improves outcomes in patients with CLL. Am Health Drug Benefits. 2015;8:39.

37. Moss HA, Chen L, Hershman DL, Davidson B, Wright JD. Adherence to PARP inhibitor therapy among women with ovarian cancer. Gynecol Oncol. 2021. https://doi.org/10.1016/j.ygyno.2021. 08.025 .

38. Friedlander ML, Moore K, Colombo N, et al. Maintenance olaparib for patients (pts) with newly diagnosed, advanced ovarian cancer (OC) and a BRCA mutation (BRCAm): 5-year (y) follow-up (f/u) from SOLO1. Ann Oncol. 2020;31(Suppl 6): S1334 (abst 2340).

39. Poveda A, Floquet A, Ledermann JA, et al. Final overall survival (OS) results from SOLO2/ENGOTov21: a phase III trial assessing maintenance olaparib in patients (pts) with platinum-sensitive, relapsed ovarian cancer and a BRCA mutation. J Clin Oncol. 2020;38(15 Suppl): abst 6002.

40. Friedlander M, Matulonis U, Gourley C, et al. Longterm efficacy, tolerability and overall survival in patients with platinum-sensitive, recurrent highgrade serous ovarian cancer treated with maintenance olaparib capsules following response to chemotherapy. Br J Cancer. 2018;119:1075-85.

41. Azmi S, Allaire J, Balk M, Dennison J, Tang B. Healthcare costs and resource utilization associated with PARP inhibitors in ovarian cancer patients initially treated with platinum-based therapy. Value Health. 2020;23(Suppl 2): S437 (abst PCN86).

42. Pal S, Gong J, Mhatre SK, et al. Real-world treatment patterns and adverse events in metastatic renal cell carcinoma from a large US claims database. BMC Cancer. 2019;19:548.

43. Shah A, Shah R, Kebede N, et al. Real-world incidence and burden of adverse events among nonmetastatic prostate cancer patients treated with secondary hormonal therapies following androgen deprivation therapy. J Med Econ. 2020;23:330-46.

44. Hansen RN, Hackshaw MD, Nagar SP, et al. Health care costs among renal cancer patients using pazopanib and sunitinib. J Manag Care Spec Pharm. 2015;21:37-44, a-d.

45. Hansen RN, Ramsey SD, Lalla D, et al. Identification and cost of adverse events in metastatic breast cancer in taxane and capecitabine based regimens. Springerplus. 2014;3:259. 